\title{
Inside the failure mechanism of tin oxide as anode for sodium ion batteries
}

\author{
Daniele Spada ${ }^{1} \cdot$ Stefania Davino ${ }^{1} \cdot$ Alessandro Girella $^{1} \cdot$ Chiara Milanese $^{1} \cdot$ Marcella Bini $^{1}$ (i)
}

Received: 7 January 2021 / Revised: 8 February 2021 / Accepted: 12 February 2021 / Published online: 24 February 2021

(C) The Author(s) 2021

\begin{abstract}
The conversion-alloying compounds have been identified as promising anode materials for sodium ion batteries (SIBs). One of them, $\mathrm{SnO}_{2}$, with an enormous theoretical capacity of $1558 \mathrm{mAh} \mathrm{g}^{-1}$ is an interesting candidate, also due to its low cost, environmental friendliness and wide availability of tin. However, many drawbacks limit its application in commercial batteries. In this paper, $\mathrm{SnO}_{2}$ has been synthesized from cheap reagents by using simple and easily scalable coprecipitation synthesis routes obtaining nanoparticles with sizes between 2 and $14 \mathrm{~nm}$ with almost spherical morphologies. The reasons of the failure of the alloying/de-alloying process were investigated by combining the results obtained from common electrochemical techniques, providing useful examples for the investigation of every material with analogous electrochemical features. Thanks to cyclic voltammetry, different reaction paths were detected for the two samples. The first cycle irreversibility was well characterized with electrochemical impedance spectroscopy, showing interesting trends in the values of the resistance. Galvanostatic cycling with potential limitations was employed to quantify the irreversibility, finding out that the most crystalline sample reached the terminal phase in the $\mathrm{Sn}-\mathrm{Na}$ system $\left(\mathrm{Na}_{15} \mathrm{Sn}_{4}\right)$, while the least crystalline sample could not achieve such a result $\left(\mathrm{Na}_{3} \mathrm{Sn}\right)$. The crystallinity of $\mathrm{SnO}_{2}$ was determined to be a key parameter, often neglected, for the realization of satisfactory anode compounds.
\end{abstract}

Keywords Sodium ion batteries $\cdot \mathrm{SnO}_{2} \cdot$ Anodes $\cdot$ Cyclic voltammetry $\cdot$ Electrochemical impedance spectroscopy $\cdot$ Galvanostatic cycling with potential limitation

\section{Introduction}

The shortage of lithium sources and their unequal distribution on the Earth contribute to increase the costs of lithium ion batteries (LIBs), also making not feasible their use for largescale energy storage systems, such as electric vehicles, currently widely required. It is urgent to find an alternative and post lithium technologies seem to be the solution $[1,2]$. Sodium ion batteries (SIBs), thanks to the sodium abundancy, low cost and high energy density, are considered promising systems even if a commercialization of them is far, mainly due to poor cycling performance and not optimized energy density. The search of suitable cathode and anode materials can benefit from the great advances done in the LIBs field in the

Marcella Bini

bini@unipv.it

1 Department of Chemistry, University of Pavia, viale Taramelli 16, 27100 Pavia, Italy last 30 years, resulting in an accelerated knowledge development process $[3,4]$. For what concerns the anode compartment, many compounds were proposed such as carbon materials, alloy-kind materials, semiconducting oxides and chalcogenides. Hard carbons are widely studied, but their reversible capacity is lower than $300 \mathrm{mAh} \mathrm{g}^{-1}$. The increase of charge storage capacity could be obtained by using materials based on energy storage mechanisms different from the classical insertion, such as alloying, conversion or both alloying/conversion. Tin-based materials attracted attention mainly due to their high theoretical capacities. Metallic tin, tin alloys or tin chalcogenides (such as sulphide or oxides) were all investigated [5]. However, Sn metal, although could alloy with sodium with a theoretical capacity of $847 \mathrm{mAh} / \mathrm{g}$, suffers of large volume changes during sodiation/de-sodiation which produce electrode pulverization, hindering its practical application. The nanostructuring process seems promising to improve the long-term stability of the anode, but currently its use is yet a challenge $[5,6] . \mathrm{SnO}_{2}$ is more attractive than $\mathrm{Sn}$ because, thanks to a conversion-alloying process, it can reach an impressive theoretical capacity of $1558 \mathrm{mAh} / \mathrm{g}$ [7-9]. The $\mathrm{SnO}_{2}$ 
storage mechanism was studied via in situ transmission electron microscopy with SAED patterns combined with density functional theory calculations and the discharge profile was divided into three regions and properly described, as well accepted in the literature $[5,7]$.

The $\mathrm{Na}_{2} \mathrm{O}$ matrix is expected to prevent the agglomeration and limit the volume expansion of tin during the alloying process, that is however the major challenge that produces fast capacity fading, as well as low conductivity, resulting in inferior rate capability.

To solve these problems, some strategies have been proposed, such as the morphology control to reduce the Na diffusion paths between electrolyte and electrode, the production of heterostructures with other semiconducting materials and the hybridization with conductive additives for reduction of volume changes and improvement of conductivity. Composites with rGO, graphene, MWCNTs or porous carbon nanofibers or with doped carbon sources, heterostructures with sulfide $\left(\mathrm{SnS}_{2}\right.$ or $\left.\mathrm{MoS}_{2}\right)$ were prepared as well as the use of oxygen vacancies engineering has been reported [10-15]. However, in the literature, not many efforts have been devoted to pure $\mathrm{SnO}_{2}$, to tailoring its particle dimensions to improve its cycling performances. In addition, the use of simple and easily available electrochemical techniques for the deep understanding of its eventual failure in the cell is often neglected.

In this paper, $\mathrm{SnO}_{2}$ was prepared from a precipitation method in water or methanol solution and characterized from structural and microscopic point of view. Then, cyclic voltammetry, electrochemical impedance spectroscopy and galvanostatic charge-discharge cycling were applied in order to investigate the involved alloying/conversion process and discuss the related failure mechanism.

\section{Experimental}

\section{Synthesis}

Two samples of $\mathrm{SnO}_{2}$ were synthesized by using a precipitation method starting from solution of $\mathrm{SnCl}_{4} \cdot 5 \mathrm{H}_{2} \mathrm{O}$ (Aldrich, $99 \%$ ) in two different solvents.

1. The first one was synthesized from an aqueous solution. The initial $\mathrm{pH}$ was very acidic, approximately one, due to the hydrolysis of tin chloride to $\mathrm{HCl}$. The solution was stirred vigorously, slowly adding a solution of $\mathrm{NaOH}$ (Merck) $5 \mathrm{M}$, until neutral $\mathrm{pH}$, which ensures that the equilibrium is shifted towards the products $\mathrm{Sn}(\mathrm{OH})_{4}$ and $\mathrm{NaCl}$. The tetra-coordinated hydroxide is insoluble and can be isolated, while the hexacoordinated hydroxide anion $\left[\mathrm{Sn}(\mathrm{Cl})_{6}\right]^{2-}$, that can be obtained at higher $\mathrm{pH}$ values, forms a soluble salt. The precipitate was centrifuged at
$6000 \mathrm{rpm}$ for $5 \mathrm{~min}$ and washed several times with water, to remove the residual $\mathrm{NaCl}$, then dried at $70^{\circ} \mathrm{C}$ for $5 \mathrm{~h}$ to convert the hydroxide into oxide.

2. The second sample was obtained from a methanol (Sigma-Aldrich) solution. $\mathrm{NH}_{3} \mathrm{OH}$ was added as basifying agent (up to $\mathrm{pH} 8-9$ ) and a final thermal treatment, after that in oven at $70^{\circ} \mathrm{C}$ for $5 \mathrm{~h}$ was performed at $500{ }^{\circ} \mathrm{C}$ for $2 \mathrm{~h}[16]$.

These samples will be hereafter named $\mathrm{SnO} 2-70$ and $\mathrm{SnO} 2-500$.

\section{Characterization}

X-ray diffraction (XRD) measurements were performed on a Bruker D5005 diffractometer, $(\mathrm{CuK} \alpha$ radiation, angular range 15-95 , step size $0.05^{\circ}$ and $15 \mathrm{~s} /$ step of counting time) in order to demonstrate the samples purity. The Rietveld method [17] was applied on the basis of the known structural model of the tin(IV) oxide, known as cassiterite. Lattice parameters, crystallite sizes, displacement error, background coefficients, atom coordinates and thermal factors were allowed to vary. The peak shape modelling, very important to obtain reliable crystallite sizes values, was performed by means of the FP (fundamental parameters) function, that takes into consideration the instrumental parameters.

Scanning electron microscopy (SEM) measurements were performed with a Zeiss EVO MA10 microscope on powder samples, sputtered with gold. Micrographs at different magnifications were also collected on the electrode pre- and postelectrochemical measurements, after washing the electrodes with acetone.

The slurries were obtained by mixing the active material with carbon (Super C65, Imerys) and PVdF in a weight ratio of 70:20:10 in N-methyl-2-pyrrolidone (NMP, Aldrich), coated on a copper foil by using a homemade doctor blade, maintained overnight at room temperature then dried in a vacuum oven at $100{ }^{\circ} \mathrm{C}$ for $1 \mathrm{~h}$. Afterwards, the slurries were hot pressed and electrodes were cut in form of discs (diameter 1 $\mathrm{cm}$ ) before being tested in Swagelok cells assembled in a dry box under argon atmosphere (MBraun, $\mathrm{O}_{2}<1 \mathrm{ppm}, \mathrm{H}_{2} \mathrm{O}<1$ ppm) using the prepared slurries as working electrode, $\mathrm{Na}$ metal as the reference and counter electrode and a Whatman GF/D disc as the separator. The electrolyte was $1 \mathrm{M} \mathrm{NaPF}_{6}$ in propylene carbonate.

Cyclic voltammetry (CV) was performed by using an Autolab PGSTAT30 (Eco Chemie) for five cycles at a scan rate of $0.1 \mathrm{mV} / \mathrm{s}$ in the potential range $0.01-3 \mathrm{~V}$. The electrochemical impedance spectroscopy (EIS) measurements were performed at room temperature by means of a Frequency response analyzer (FRA) Autolab PGSTAT30 apparatus in the frequency range $0.01-10^{6} \mathrm{~Hz}$, at the $\mathrm{OCV}$, at the beginning and at the end of charge and discharge (0.01 and $3 \mathrm{~V})$. 
Galvanostatic cycling with potential limitation (GCPL) tests were performed on a Neware Battery Test System in the 0.01$3 \mathrm{~V}$ potential range at $\mathrm{C}$ rates between $\mathrm{C} / 20$ and $\mathrm{C} / 2$.

\section{Results and discussion}

In the following, the two samples will be characterized from both a structural and morphological point of view, before being studied with simple and easily available electrochemical techniques.

\section{Structure and morphology}

Both the syntheses brought to the desired product, $\mathrm{SnO}_{2}$, in the crystalline form known as Cassiterite (JCPDS N. 41-1445, isostructural with Rutile), but the different thermal treatment is considered the main cause of the difference in the XRD patterns (Fig. 1a). The further calcination at $500{ }^{\circ} \mathrm{C}$ allowed the crystallites to grow much more than the bare drying at $70{ }^{\circ} \mathrm{C}$ : in fact, the $\mathrm{SnO} 2-500$ sample shows broad but resolved peaks, while the SnO270 sample has a diffraction pattern typical of nanocrystalline samples. The Rietveld structural refinements were satisfactory for both the samples (Rwp/GoF of 5.87/1.26 and 7.75/1.65 for $\mathrm{SnO} 2-70$ and $\mathrm{SnO} 2-500$ respectively). Almost one order of magnitude difference in the crystallite sizes values ( $2 \mathrm{~nm}$ vs $14 \mathrm{~nm}$, Fig.1a) was quantified, thanks to the Rietveld refinement [17]. Notably, an expansion of the lattice parameters in the $\mathrm{SnO}-70$ sample was also found, hardly noticeable from the powder patterns due to extreme peak broadening (Fig.1a). The values of the lattice parameters for $\mathrm{SnO} 2-500$ are very close to those taken from literature $(4.7380 \AA$ and $3.1865 \AA)$, [18] suggesting that the crystallization of $\mathrm{SnO}_{2}$ involves a contraction of the lattice parameters.

The particles are constituted by almost isotropic aggregates of $10 \mu \mathrm{m}$ of smaller sub-micrometric particles, with some obvious edges, as determined from SEM measurements (Fig. $1 \mathrm{~b}$ and $1 \mathrm{c}$ for $\mathrm{SnO} 2-70$ and $\mathrm{SnO} 2-$ 500 respectively). The two samples do not show substantial differences.
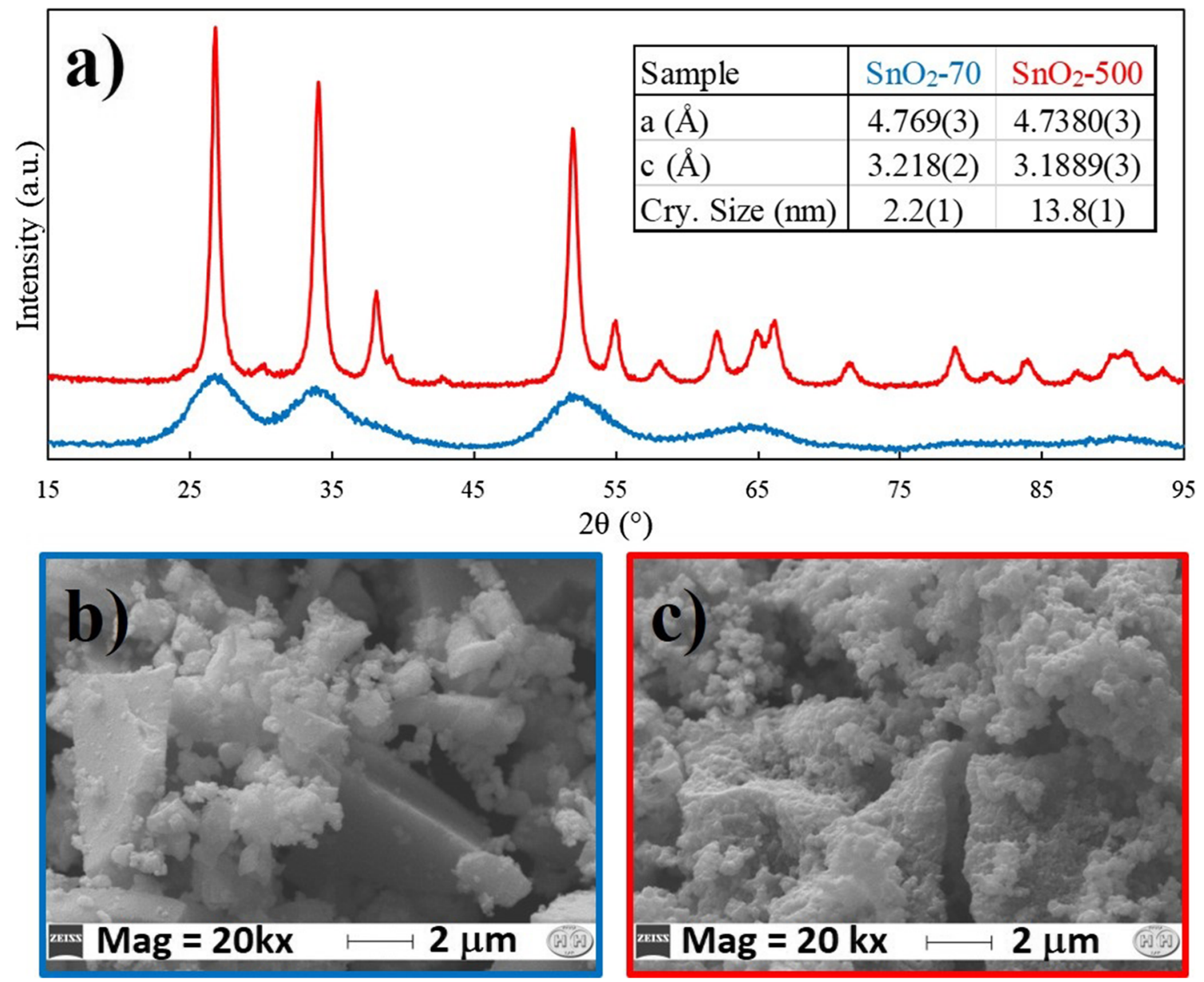

Fig. 1 XRD patterns of SnO2-70 (blue) and SnO2-500 (red), along with the corresponding refined lattice parameters and crystallite sizes (a), SEM images of $\mathrm{SnO} 2-70(\mathbf{b})$ and $\mathrm{SnO} 2-500$ (c) samples 


\section{CV}

Cyclic voltammetry is a very useful tool in order to study the electrochemistry of conversion-alloying reactions. In a monophasic sample, the sequence of the reactions is a certain way to attribute the current peaks. The multi-step reaction proceeds necessarily from the conversion to the formation of progressively more sodiated alloys. The two micron-sized samples $\mathrm{SnO} 2-70$ and $\mathrm{SnO} 2-500$ were tested with a simple carbonate-based electrolyte without additives, which is a cheap choice. These can be considered the perfect conditions for a conversion-alloying anode to fail after a few cycles. It is well known indeed that conversion-alloying materials suffer from severe structural changes upon cycling associated with large volume expansion and consequent electrode disintegration and capacity fading. The freshly exposed electrode material upon every alloying cycle implies a continuous electrolyte reduction, strongly affecting the overall coulombic efficiency and cycle life [3].

The $\mathrm{Sn}-\mathrm{Na}$ phase diagram at room temperature consists of the following phases: $\mathrm{NaSn}_{6}, \mathrm{NaSn}_{4}, \mathrm{NaSn}_{3}, \mathrm{NaSn}_{2}, \mathrm{Na}_{9} \mathrm{Sn}_{4}$, $\mathrm{Na}_{3} \mathrm{Sn}$ and $\mathrm{Na}_{15} \mathrm{Sn}_{4}$. The crystal structure of $\mathrm{Na}_{9} \mathrm{Sn}_{4}$ is orthorhombic and that of $\mathrm{Na}_{15} \mathrm{Sn}_{4}$ is cubic, whereas the others are not fully established. For pure $\mathrm{Sn}$ and high $\mathrm{Sn}$ content alloys, transmission electron microscopy (TEM) [5, 7] shows the $\mathrm{Na}_{15} \mathrm{Sn}_{4}$ phase forms as generally spherical nanoparticles, whereas in alloys rich in other elements, more complex flake-like morphologies are observed. The sequence of the reactions observed from SAED patterns obtained from an in situ TEM study $[5,7]$ is:

I. $\mathrm{SnO}_{2}+4 \mathrm{Na}^{+}+4 \mathrm{e}^{-} \leftrightarrow \mathrm{Sn}+2 \mathrm{Na}_{2} \mathrm{O}$
II. $\mathrm{Sn}+\mathrm{xNa}^{+}+\mathrm{xe}^{-} \leftrightarrow \mathrm{Na}_{\mathrm{x}} \mathrm{Sn} x<0.5$,

III. $\mathrm{Na}_{\mathrm{x}} \mathrm{Sn}+\mathrm{yNa}^{+}+\mathrm{ye}^{-} \leftrightarrow \mathrm{NaSn}_{2} \quad y=0.5-x$

IV. $\mathrm{NaSn}_{2}+7 / 4 \mathrm{Na}^{+}+7 / 4 \mathrm{e}^{-} \leftrightarrow \mathrm{Na}_{9} \mathrm{Sn}_{4}$

V. $\mathrm{Na}_{9} \mathrm{Sn}_{4}+3 / 4 \mathrm{Na}^{+}+3 / 4 \mathrm{e}^{-} \leftrightarrow \mathrm{Na}_{3} \mathrm{Sn}$

VI. $\mathrm{Na}_{3} \mathrm{Sn}+3 / 4 \mathrm{Na}^{+}+3 / 4 \mathrm{e}^{-} \leftrightarrow \mathrm{Na}_{15} \mathrm{Sn}_{4}$

The conversion reaction (step I) brings $711 \mathrm{mAh} \mathrm{g}^{-1}$ but does not create bonds between $\mathrm{Sn}$ and $\mathrm{Na}$, creating instead insulating sodium oxide. The overall reversible alloying (steps II-VI) bring $847 \mathrm{mAh} \mathrm{g}^{-1}$. The experimental positions of voltage plateaus did not directly correlate to ab initio modelling of sodiating Sn, which is expected both due to the uncertainty in the equilibrium structure of the intermediate phases, and the kinetic difficulty of nucleation at room temperature. It is important to note that the reaction sequence and crystal structures observed even in the $100-\mathrm{nm}$ films on which
TEM measurements were performed $[5,7]$ could be different at higher charging rates and/or even finer microstructures. For instance, a $1.9-\mathrm{V}$ plateau that is present at low currents is absent at high currents and the phase sequence also depends on the terminal cut-off voltage [5].

Following these premises, the attribution of the peaks in Fig. 2 a (the comparison between the first CV cycle of both samples) can be hypothesized: the conversion reaction is thought to take place in the range $1.5-1.0 \mathrm{~V}$ (Fig. 2b). The peak at about $1.0 \mathrm{~V}$ is attributed to the formation of alloys with low sodium content $\left(\mathrm{Na}_{\mathrm{x}} \mathrm{Sn}\right)$, and the others at 0.7, 0.4 and $0.3 \mathrm{~V}$ correspond to the formation of $\mathrm{NaSn}_{2}, \mathrm{Na}_{9} \mathrm{Sn}_{4}$ and $\mathrm{Na}_{3} \mathrm{Sn}$, respectively, while the peak at the cut-off voltage is related to the terminal intermetallic phase $\mathrm{Na}_{15} \mathrm{Sn}_{4}$. During the oxidative branch, the situation is much less clear, preventing a meaningful peak attribution.

The relative ratio of the peak currents suggests different reaction paths for the two samples. For the $\mathrm{SnO} 2-70$, the most important reaction seems to be the formation of the $\mathrm{Na}_{3} \mathrm{Sn}$ alloy, while for $\mathrm{SnO} 2-500$, the predominant is the formation of the terminal alloy $\mathrm{Na}_{15} \mathrm{Sn}_{4}$. This can be taken as an indication of a better reactivity of the $\mathrm{SnO} 2-500$ sample towards sodium, while the reaction stops sooner for the $\mathrm{SnO} 2-70$ sample. During charge, many differences are also evident, but an exact attribution is hindered by the exceptional broadening of the peaks. A high overpotential (anodic peaks start from 0.2 V) and a low coulombic efficiency can be easily observed by the position and intensity of anodic peaks, compared to the corresponding cathodic ones.

After the first cycle, the voltammograms change markedly (Fig. 2c, d), with a pronounced broadening even in the cathodic scan. All the peak intensities decrease cycle after cycle, indicating progressively slower reaction rates, and also the cathodic peak intensity ratios change markedly: in both samples, the highest peak is the formation of the terminal alloy after the first cycle, with all the other peaks flattening to a sloping region below $1 \mathrm{~V}$. The reaction seems to proceed more homogeneously after the first cycle, with less marked concentration gradients in the particles of active material.

\section{EIS}

Electrochemical impedance spectroscopy has a great potential for studying electrochemical cells. Thanks to the sweep in the excitation frequencies, the different charge transfer reactions (CTRs) can be studied separately in detail. By applying the excitation signal to different steady state voltages, the evolution of the reaction can be characterized as a function of the cycles.

The results of the EIS measurements are reported in Fig. 3; the spectra were modelled as one or two charge transfer resistances (depending on the data), and neglecting the mass transport (Fig. S1 and Table S1 in the Supporting Information). 

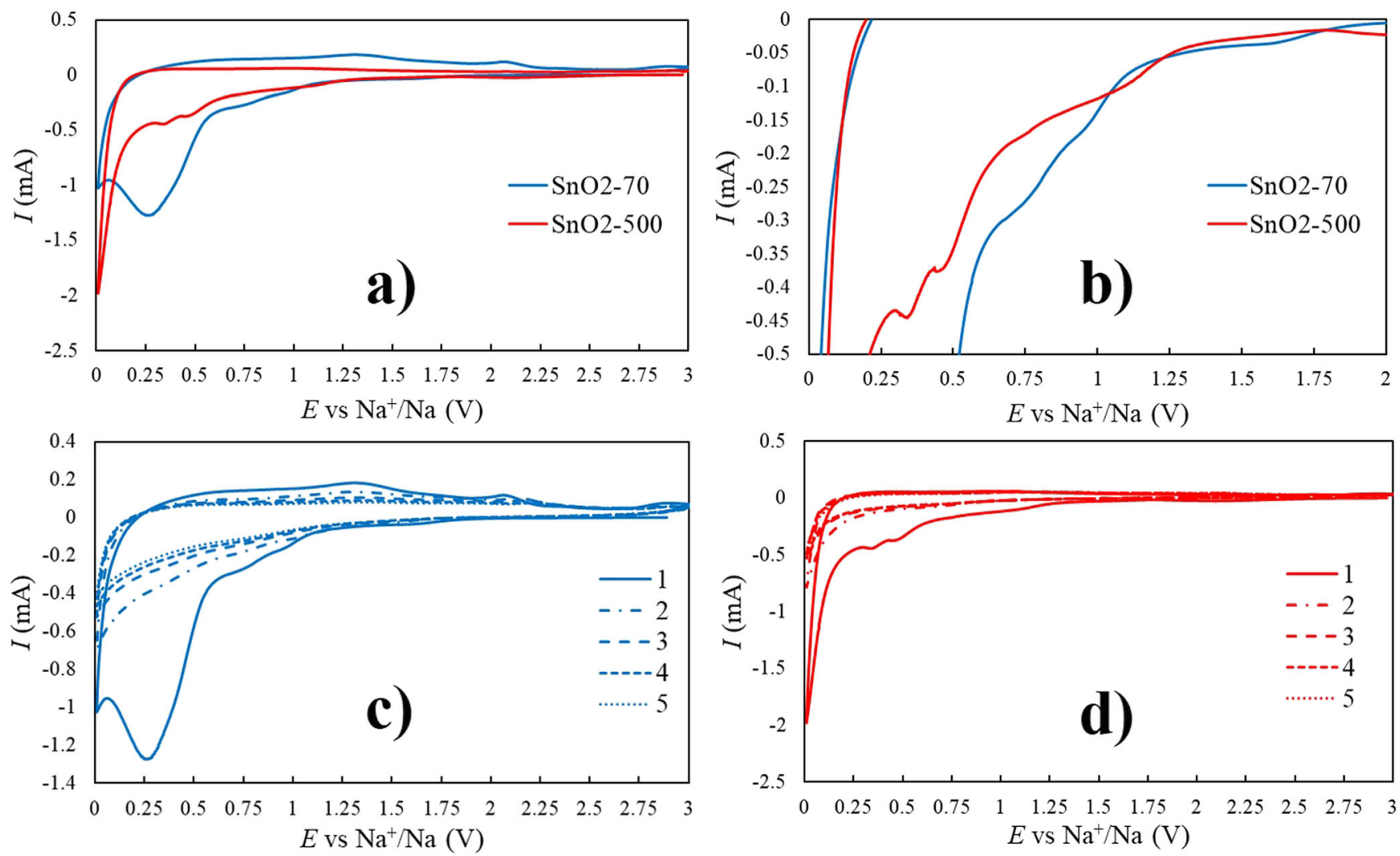

Fig. 2 Voltammograms for a) the first cycles of both samples with b) greater detail on lower peaks; comparison between the first and the following cycles for c) $\mathrm{SnO} 2-70$ and d) $\mathrm{SnO} 2-500$

For the $\mathrm{SnO}_{2}-\mathrm{Sn}-\mathrm{Na}_{\mathrm{x}} \mathrm{Sn}$ system, in OCV conditions (approximately $3 \mathrm{~V}$ ), the system shows a broad semicircle, hard to model, followed by a straight line (not a conventional Warburg impedance, because of the higher slope) (Fig. 3) [19], with the electrons involved in the charge transfer reaction (CTR). There is no doubt about the electronic nature of the $\mathrm{CTR}$, because its frequency was determined to be higher than $10^{3} \mathrm{~Hz}$ (Fig. 3c, f). The high values of the resistance in Fig. 3 a and d (> $1000 \Omega$ notwithstanding $20 \%$ wt of carbon addition) can be explained with the semi conductive nature of $\mathrm{SnO}_{2}$, the starting active material. At $0.01 \mathrm{~V}$, the discharge cut-off voltage (Fig. 3b, e), the active material is transformed into a $\mathrm{Sn}-\mathrm{Na}$ alloy, with metallic conductivity. The CTR of electrons is favoured, with an order of magnitude drop in the associated resistance (Tab. S1), while another CTR of $\mathrm{Na}^{+}$ions should appear at much lower frequencies, alongside with mass transport, but a proper detection of the phenomenon is prevented by the instrumental limit $(0.01 \mathrm{~Hz})$. This means that the charge transfer of $\mathrm{Na}^{+}$ions to Na-Sn alloys is slow and highly resistive, confirming the kinetic issues associated with this reaction. In the literature, for nano-sized samples, the same CTR is often detected, but nanostructured Sn can promote the homogeneous sodiation/de-sodiation in a single particle and alleviate the volume mismatch, thus avoiding crack propagation and improving structural stability [6].
The values of electronic resistance in non-OCV conditions (Fig. 4) are still quite high for an alloy (metals and alloys tend to show values in the range of tens of Ohms), but they can be explained with the surrounding insulating $\mathrm{Na}_{2} \mathrm{O}$ formed during the conversion reaction. The irreversibility of the reaction is manifested in the 3-V spectrum, which differs from its analogous (OCV) not only in the values, but also in the shape (Fig. $3 \mathrm{~b}, \mathrm{e})$. The value of the electronic CTR is still very low, confirming the irreversibility of the conversion reaction, and an ionic CTR can be detected even though at $3 \mathrm{~V}$ the active material should be completely de-sodiated. This confirms that the alloy did not even de-sodiate to Sn metal, but instead regressed to some lower stoichiometry alloy. The detection of the ionic CTR indicates a much faster reaction involving sodium ions, suggesting that sodium atoms can diffuse faster in lower stoichiometries alloys.

It has to be noted that no further resistances appear after the 1st discharge, supporting the absence of a SEI layer that should have formed after the reductive decomposition of the electrolyte.

The evolution of the resistance values with the cycles (Fig. 4) shows increasing trends that can be explained with the analogous cycles of mechanical stresses due to severe expansion/contraction cycles that negatively affect the mechanical properties of the electrode, but also with the 

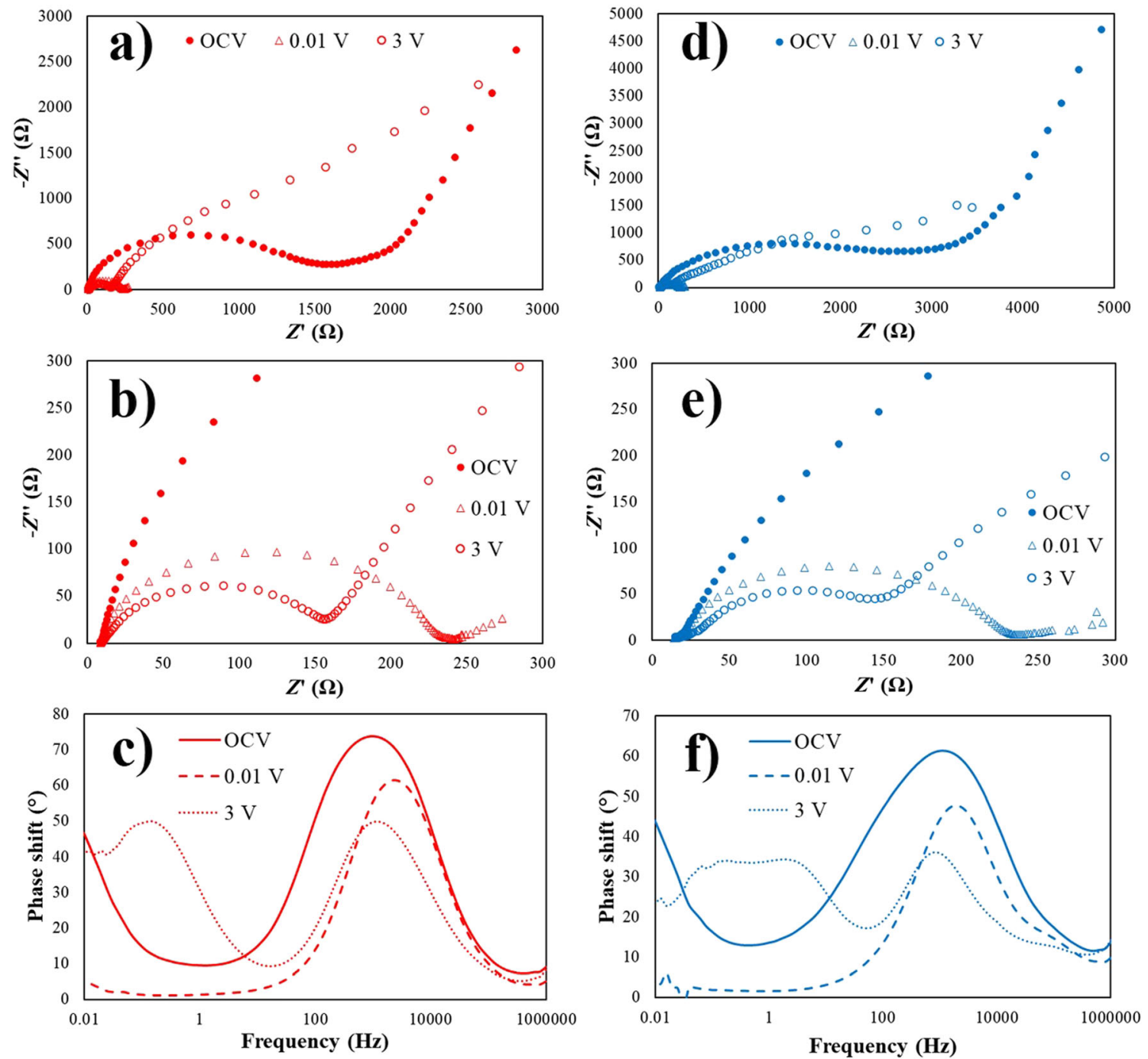

Fig. 3 EIS spectra of the 1st cycle of charge and discharge, for $\mathrm{SnO} 2-500(\mathbf{a}-\mathbf{c})$ and $\mathrm{SnO} 2-70(\mathbf{d}-\mathbf{f})$ samples. The (b) and (e) graphs are the enlargement of the high frequency region of the relative (a) and (d) plots. Both Nyquist plot (a, b and $\mathbf{d}, \mathbf{e})$ and Bode phase plot (c, f) are shown.

irreversibility of the reaction. As will be discussed in the next section, the formation of a Na-Sn alloy in the first discharge is not reversible, being the de-sodiation very little (as observed also by the currents in the anodic branch of the $\mathrm{CV}$ ). The sodiation degree slowly increases in the later cycles, and this brings to a sample that gets gradually more sodiated.

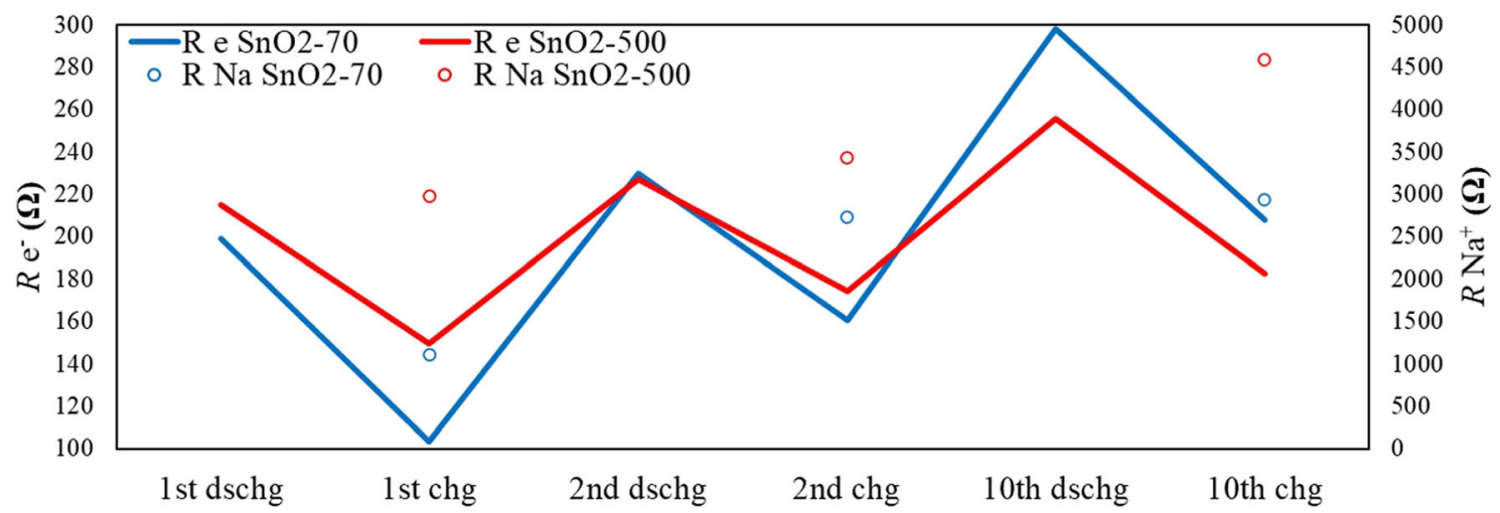

Fig. 4 Electronic (lines) and ionic (empty dots) charge transfer resistances with cycling, for both samples 
Simulations reported in the literature [20] demonstrated that sodiation of Sn leads to a 75\% decrease in its Young's and shear modulus, due to a progressive increase of $\mathrm{Na}$ near neighbours to $\mathrm{Sn}$, substituting weaker $\mathrm{Sn}-\mathrm{Na}$ bonds for $\mathrm{Sn}-\mathrm{Sn}$ bonds. This has several important implications for the microstructure, mechanical properties and cycling performance of sodiated Sn: the tremendous drop in the modulus affects the elastic response of the $\mathrm{Sn}$ to the stresses associated with volumetric changes during charging/discharging, both intrinsic (inside the material due to nonuniform sodiation) and extrinsic (mismatch with current collector or other phases) [7].

Even if the values of electronic resistances are comparable between the samples, the higher values of ionic resistance shown by $\mathrm{SnO} 2-500$ seems to suggest a higher sodium content in the electrode with respect to $\mathrm{SnO} 2-70$.

\section{GCPL}

Charge-discharge measurements, also known as galvanostatic cycling with potential limitation, stand at the basis of the characterization of every electrode for secondary batteries, and are so common that they cannot be excluded from any electrochemical paper [21]. They allow to directly measuring the charge and discharge capacities that can be obtained from the application of a constant current between the electrodes, as a function of the number of cycles. From the same data, also the coulombic efficiency and the capacity retention can be calculated, indicating the reversibility of the reaction in the same cycle and along the cycles, respectively.

During the rate capability test (testing the electrode response to increasing values of current), the $\mathrm{SnO} 2-500$ sample overperformed $\mathrm{SnO} 2-70$ in the 1st cycle (Table in Fig. 5), but with half the coulombic efficiency. Thereafter, both the capacity retention and even the coulombic efficiency of SnO2-500 are higher, indicating better reversibility of the reaction (Fig. $5)$. The capacity values are very low for both samples, as expected for such a kinetically slow reaction in micron-sized samples. The coulombic efficiency sharply increases after the first cycle (80-90\%), reaching a sort of plateau after 10-20 cycles, depending on the sample.

All these simple observations point towards a difficult reaction, especially during the first charge. This suggests that when the particles of Sn get sodiated, the de-sodiation is not complete. Then, the sodiation of the second cycle starts from already sodiated particles, which are less prone to further sodiation, due to less residual sites for Sodium storage. This process goes on cycle after cycle, until a sort of stationary state where the (small) amount of sodium alloyed during discharge equals the amount of sodium extracted during charge.

As mentioned above, the first electrochemical reaction in a $\mathrm{SnO}_{2}$-containing electrode is the conversion reaction to $\mathrm{Na}_{2} \mathrm{O}$ and Sn. In the voltage profile (Fig. 6, left), the corresponding capacity can be calculated from the cumulative capacity obtained at $1 \mathrm{~V}$. Taking into account the correction of the 1st cycle capacity (Fig. 6), when electrons are consumed in the conversion reaction but not alloyed, a quantitative correlation between the capacity and the sodium amount in the electrode can be established. The correction was performed only in the first cycle because the EIS measurements did not support the hypothesis of a reversible conversion reaction. The graph shows the data trend of GCPL measurements, in a clearer way: each point after the 1st was calculated by the simple rule of subtracting the charge capacity from the discharge capacity (odd steps are discharges, and even steps are charges). By this way, with a simple data transformation, the irreversibility of the reactions can be easily discussed: the data (Fig. 6, right) tend towards a plateau that indicates the maximum amount of sodium that can be stored into the electrode.

SnO2-500 stores a higher amount of sodium/f.u. than SnO2-70, with very little reversibility: the coulombic efficiency is correlated to the oscillation between charges and discharges. This confirms the presence of residual alloys when the electrode is fully charged observed with EIS measurements, but also the higher sodium content deduced before.

The main issue of the electrodes seems to be the 1st discharge reaction, as already observed from the 1 st cycle coulombic efficiency, but we have the confirmation of what hypothesized from $\mathrm{CV}$ measurements i.e. the different reaction paths for the 2 samples. SnO2-500 reaches the highest stoichiometry of 3.75 atoms of $\mathrm{Na}$ per $\mathrm{Sn}$ while the $\mathrm{SnO} 2-70$ sample only reaches the less sodiated phase, with 3 atoms of $\mathrm{Na}$ per $\mathrm{Sn}$. This could be taken as a proof of the importance of the crystallinity in $\mathrm{SnO}_{2}$ electrodes for $\mathrm{Na}$ storage.

\subsection{Post-mortem SEM}

In conversion-alloying systems, the expansion-contraction cycles induce severe stresses in the electrode, which must exhibit excellent mechanical properties to avoid fractures and pulverization, dispersion of particles of active material into the liquid electrolyte and loss of contact with the current collector. Postmortem SEM images offer a great insight into the effects of cycling on the integrity and the morphology of the electrode. Figure $7 \mathrm{a}-\mathrm{d}$ show that pulverization did not seem to occur, without evident cracks in the electrode. What is interesting is the flake-like morphology, atypical for $\mathrm{Na}_{15} \mathrm{Sn}_{4}$ which is usually formed as a crystalline phase with spherical nanoparticles. In both samples, nucleation of intermetallic phases is radial from what were edgy but mostly isotropic particles of $\mathrm{SnO}_{2}$. On the basis of previous results, the edgy nucleation of $\mathrm{SnO} 2-$ 70 is thought to relate to the $\mathrm{Na}_{3} \mathrm{Sn}$ phase, while the algae-like morphology of the particles of $\mathrm{SnO} 2-500$ is believed to correspond to $\mathrm{Na}_{15} \mathrm{Sn}_{4}$ phase.

The morphology of the final particles is built during the first cycle, where particles of $\mathrm{SnO}_{2}$ are converted to $\mathrm{Sn}$ surrounded by $\mathrm{Na}_{2} \mathrm{O}$ (see the reaction mechanisms previously 
Fig. 5 Discharge capacities (full circles) and corresponding coulombic efficiencies (empty squares) for the rate capability test. The values for the first cycle are reported in the table

\begin{tabular}{|l|c|c|}
\hline & \multicolumn{2}{|c|}{ First cycle } \\
\hline Sample & Dschg. Cap. (mAh/g) & Coul. Eff. (\%) \\
\hline SnO2-70 & 679 & 31.0 \\
SnO2-500 & 907 & 15.0 \\
\hline
\end{tabular}

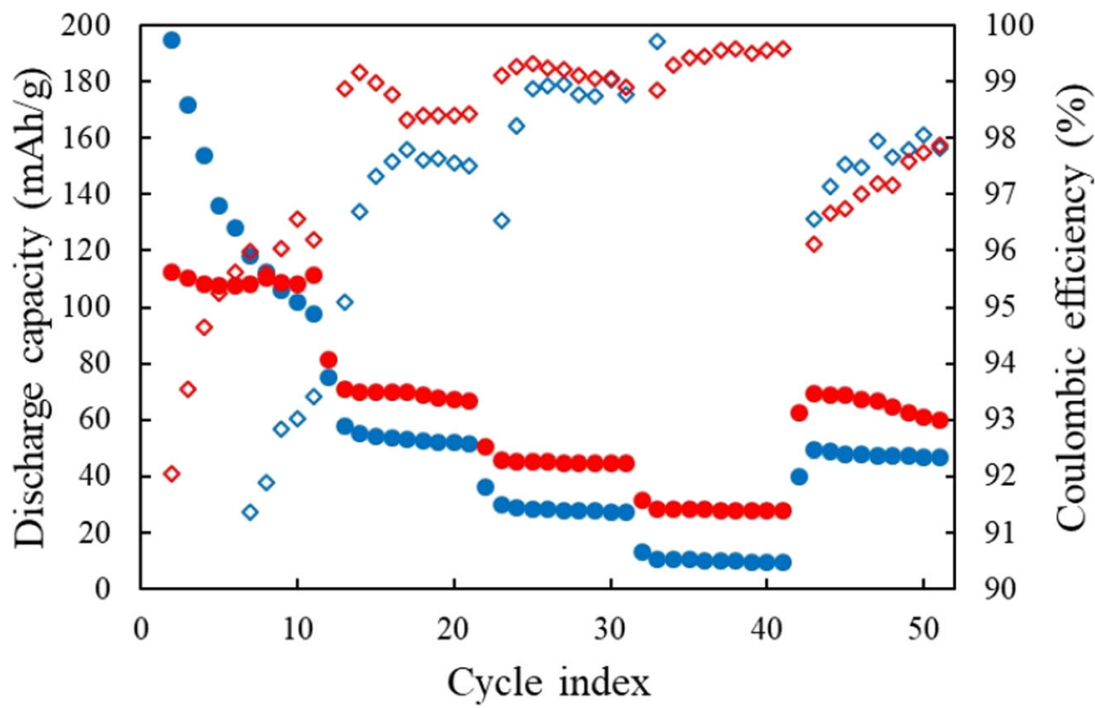

reported). Then, the morphology of Sn particles is influenced by the nucleation of different alloys, with great volume changes (compare the scale in Fig. 7 and Fig. 1).

\section{Conclusions}

We could demonstrate how very simple and widespread electrochemical techniques can be successfully employed in the explanation of failure in $\mathrm{SnO}_{2}$ employed as conversionalloying anode in SIBs. Analogous considerations can be extended to any other material with a similar behaviour e.g. other conversion-alloying materials or any other electrode undergoing irreversible reactions.

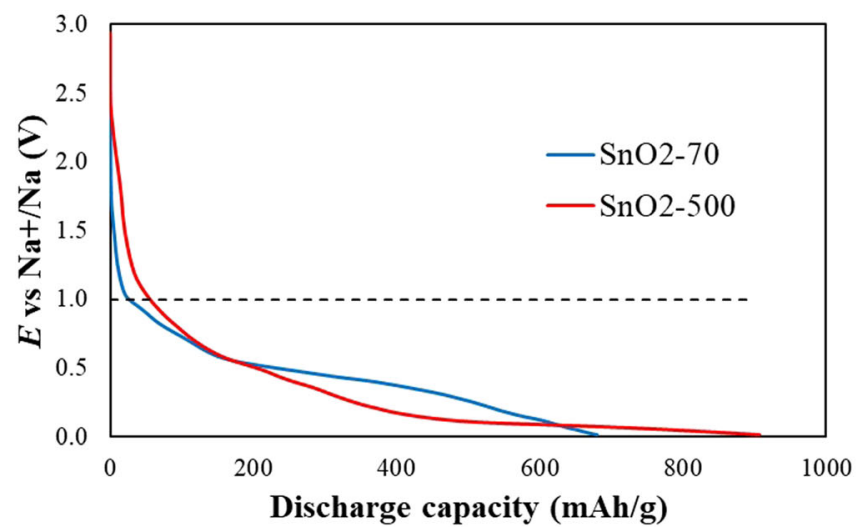

With a simple cyclic voltammetry experiment, differences in the reaction paths could be spotted, alongside hysteresis and irreversibility, very common in conversion-alloying systems.

Degradations in the electrode, absence of SEI layer and irreversibility could all be detected with the electrochemical impedance spectroscopy, through the detailed analysis of the charge transfer resistances associated with electrons and sodium ions at different potentials.

From the charge-discharge measurements, the performance of the electrode could be easily evaluated. From these clues combined together, important considerations on the reversibility and the amount of sodium stored into the electrode were inferred and supported with the help of the post-mortem SEM analysis that could picture the situation of the electrode after cycling.

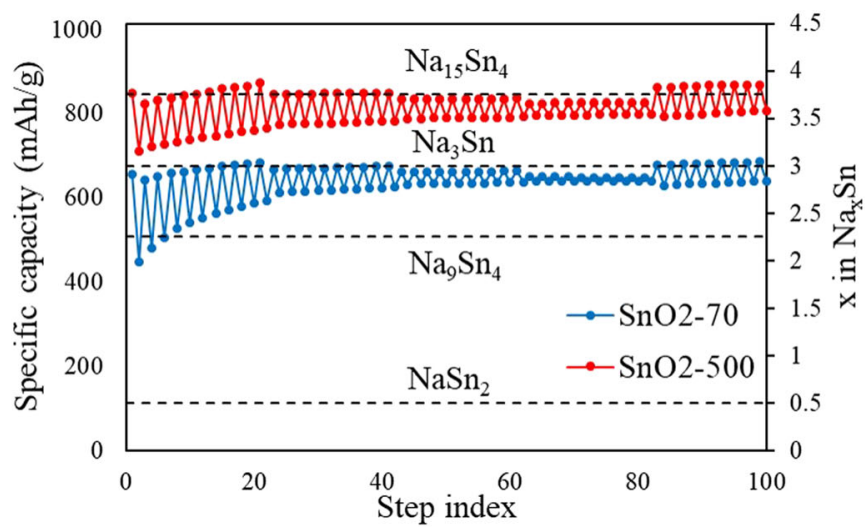

Fig. 6 Voltage profile (left) and correlation between the specific capacity and the sodium stoichiometry (right) 

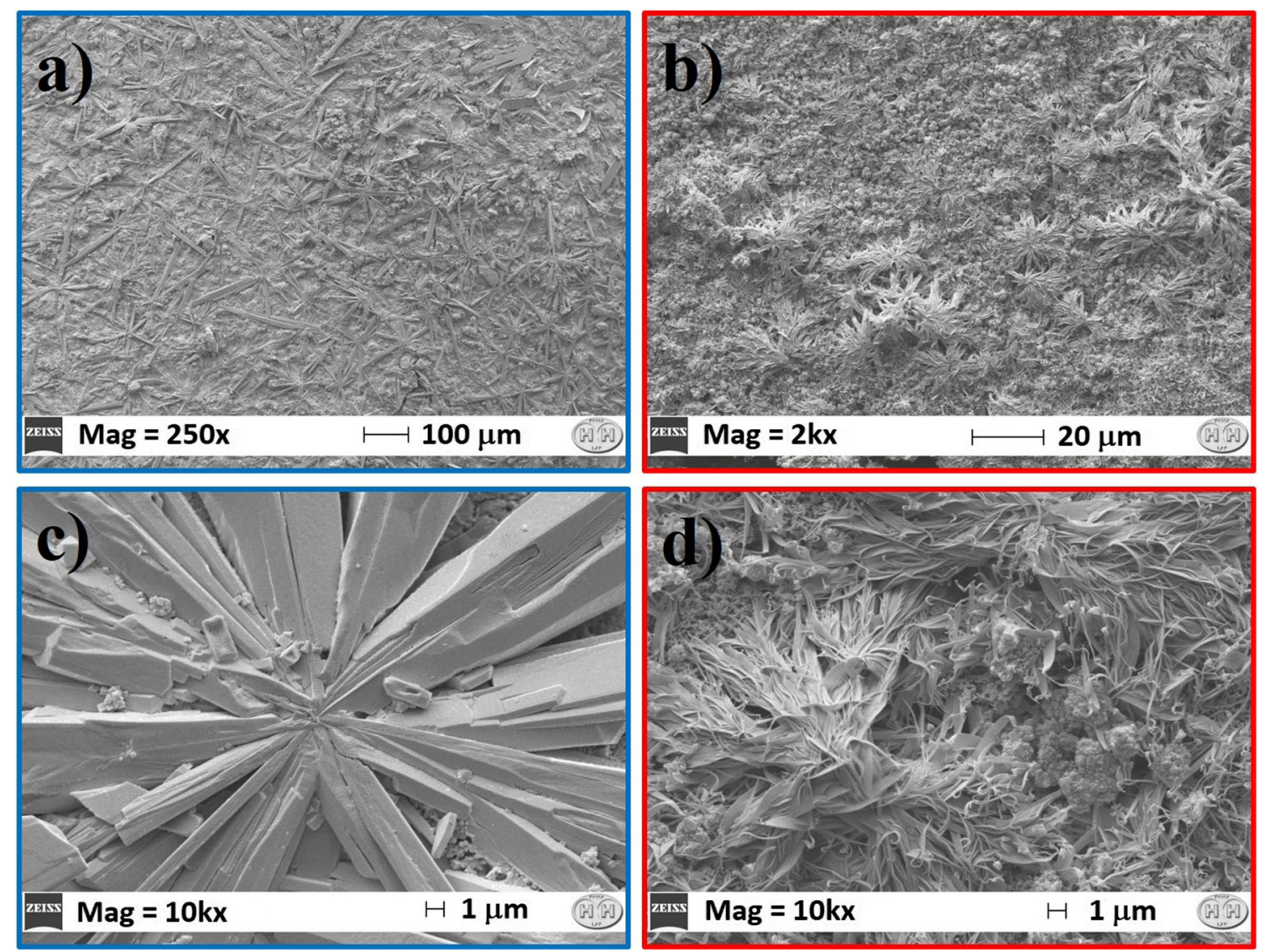

Fig. 7 Post-mortem SEM images of the electrodes containing SnO2-70 (blue) and SnO2-500 (red), at lower (a, b) and higher (c, d) magnification

In conclusion, the crystallinity of $\mathrm{SnO}_{2}$ seems to play an important (but maybe underestimated) role for the alloying reaction, allowing the storage of more sodium ions, alongside with nanostructuration, surface coating and preparation of composites that enhance the overall reversibility of the reaction.

Supplementary Information The online version contains supplementary material available at https://doi.org/10.1007/s10008-021-04919-9.

Author contribution Conceptualization: Marcella Bini, Daniele Spada; methodology: Stefania Davino; formal analysis and investigation: Stefania Davino, Daniele Spada, Chiara Milanese, Alessandro Girella; writing-original draft preparation: Daniele Spada; Writing-review and editing: Daniele Spada, Marcella Bini; supervision: Marcella Bini

Funding Open access funding provided by Università degli Studi di Pavia within the CRUI-CARE Agreement.

Data availability The datasets generated during and/or analyzed during the current study are available from the corresponding author on reasonable request.

\section{Declarations}

Conflict of interest The authors declare no competing interests.

Open Access This article is licensed under a Creative Commons Attribution 4.0 International License, which permits use, sharing, adaptation, distribution and reproduction in any medium or format, as long as you give appropriate credit to the original author(s) and the source, provide a link to the Creative Commons licence, and indicate if changes were made. The images or other third party material in this article are included in the article's Creative Commons licence, unless indicated otherwise in a credit line to the material. If material is not included in the article's Creative Commons licence and your intended use is not permitted by statutory regulation or exceeds the permitted use, you will need to obtain permission directly from the copyright holder. To view a copy of this licence, visit http://creativecommons.org/licenses/by/4.0/.

\section{References}

1. Durmus YE, Zhang H, Baakes F, Desmaizieres G, Hayun H, Yang L, Kolek M, Küpers V, Janek J, Mandler D, Passerini S, Ein-Eli Y (2020) Side by side battery technologies with lithium-ion based batteries. Adv Energy Mater 10(24):2000089

2. Placke T, Kloepsch R, Dühnen S, Winter M (2017) Lithium ion, lithium metal, and alternative rechargeable battery technologies: the 
odyssey for high energy density. J Solid State Electrochem 21(7): 1939-1964

3. Zhang H, Hasa I, Passerini S (2018) Beyond Insertion for na-ion batteries: nanostructured alloying and conversion anode materials. Adv Energy Mater 8:17

4. Sun Y, Guo S, Zhou H (2019) Exploration of advanced electrode materials for rechargeable sodium-ion batteries. Adv Energy Mater 9(23): 1800212

5. Li Z, Ding J, Mitlin D (2015) Tin and tin compounds for sodium ion battery anodes : phase transformations and performance. Acc Chem Res 48(6): 1657-1665

6. Ying H, Han W (2017) Metallic Sn-based anode materials : application in high-performance lithium-ion and sodium-ion batteries. Adv Sci 4:1700298

7. Li F, Liu Q, Hu J, Yang J, Ma J (2020) Recent progresses on $\mathrm{SnO}_{2}$ anode materials for sodium storage. J. Phys. D: Appl. Phys. 53(35): 353001

8. Zoller F, Bohm D, Bein T, Fattakhova-Rohlfing D (2019) Tin oxide based nanomaterials and their application as anodes in lithium-ion batteries and beyond. ChemSusChem 12(18):4140-4159

9. Kebede MA (2020) Tin oxide-based anodes for both lithium-ion and sodium-ion batteries. Curr Opin Electrochem 21:182-187

10. Yang Y, Pan ZZ, Wang YY, Ma YC, Li C, Lu YJ, Wu XL (2019) Ionic-liquid-bifunctional wrapping of ultrafine $\mathrm{SnO} 2$ nanocrystals into N-doped graphene networks: high pseudocapacitive sodium storage and high-performance sodium-ion full cells. Nanoscale 11(31):14616-14624

11. Kong Z, Liu X, Wang T, Fu A, Li Y, Guo P, Guo YG, Li H, Zhao XS (2019) Three-dimensional hollow spheres of porous $\mathrm{SnO} / \mathrm{rGO}$ composite as high performance anode for sodium ion batteries. Appl Surf Sci 479:198-208

12. Zhang X, Liu K, Zhang S, Miao F, Xiao W, Shen Y, Zhang P, Wang Z, Shao G (2020) Enabling remarkable cycling performance of high-loading MoS2@Graphene anode for sodium ion batteries with tunable cut-off voltage. J Power Source 458:228040

13. Yang X, Xiao F, Wang S, Liu J, Leung MKH, Yu DYW, Rogach AL (2019) Confined annealing-induced transformation of tin oxide into sulfide for sodium storage applications. J Mater Chem A 7(19): $11877-11885$

14. Cen D, Ding Y, Wei R, Huang X, Gao G, Wu G, Mei Y, Bao Z (2020) Synthesis of metal oxide/carbon nanofibers via biostructure confinement as high-capacity anode materials. ACS Appl Mater Interfaces 12(26):29566-29574

15. Lu X, Mao Q, Chen Y, Bao L, Tong L, Xiong Q, Qin H, Pan H, Ji Z (2018) A novel oxygen vacancy introduced microstructural reconstruction of $\mathrm{SnO} 2$-graphene nanocomposite: Demonstration of enhanced electrochemical performance for sodium storage. Electrochim Acta 282:351-361

16. Kumar V, Singh K, Kumar A, Kumar M, Singh K, Vij A, Thakur A (2017) Effect of solvent on crystallographic, morphological and optical properties of $\mathrm{SnO} 2$ nanoparticles. Mat Res Bull 85:202-208

17. Coelho AA (2009) Bruker AXS TOPAS Version 4.2

18. Baur WH, Khan AA (1971) Rutile-type compounds. IV. SiO2, $\mathrm{GeO} 2$ and a comparison with other rutile-type structures. Acta Cryst B 27(11):2133-2139

19. Randles JEB (1947) Kinetics of rapid electrode reactions. Discuss Faraday Soc 1:11-19

20. Mortazavi M, Deng J, Shenoy VB, Medhekar NV (2013) Elastic softening of alloy negative electrodes for Na-ion batteries. J Power Source 225:207-214

21. Yang X, Rogach AL (2019) Electrochemical Techniques in battery research: a tutorial for nonelectrochemists. Adv Energy Mater 9(25):1900747

Publisher's note Springer Nature remains neutral with regard to jurisdictional claims in published maps and institutional affiliations. 